\title{
Cluster splitting in granular segregation driven by horizontal shaking
}

\author{
Angel Garcimartín ${ }^{1, \star}$, Ignacio Larrea ${ }^{1}$, Celia Lozano ${ }^{2}$, and Iker Zuriguel ${ }^{1}$ \\ ${ }^{1}$ Departamento de Física, Facultad de Ciencias, Universidad de Navarra, E-31080 Pamplona, Spain. \\ ${ }^{2}$ Max-Planck-Institut für Intelligente Systeme, Heisenbergstraße 3, 70569 Stuttgart, Germany.
}

\begin{abstract}
In a recent work [C. Lozano et al. Phys. Rev. Lett 114, 178002 (2015)] segregation in an horizontally shaken granular layer was studied by analysing the particle-particle interactions in the simplest case possible of a two particles cluster. There, it was found that all clusters are transient (they eventually split if one waits long enough) and the probability distribution function of the separation times displays a power law tail, indicating that the splitting probability is not constant over time. Here, we extend this study to clusters of 3, 5, 10 and 20 particles where we also observe the power law decay of the distribution of cluster splitting time. In addition, we observe a weak increase of the average cluster splitting time with the cluster size, suggesting that interaction forces are non-additive. Finally, we show interesting statistics on the way in which clusters break suggesting that escaping of individual particles in the cluster borders is more likely than cluster breakage in subclusters of similar size.
\end{abstract}

\section{Introduction}

If a mixture of grains is fluidized, granular segregation may occur driven by differences in particle size, density, shape, surface properties, restitution coefficient, rolling friction, and so on [1-3]. Sometimes segregation occurs when similar particles tend to move towards a particular location. The canonical example of this is the Brazil nut effect in vertical shaking [4]. A different case is when there is not such distinct spatial affinity of any of the mixture constituents for a given place. Then, segregation necessarily occurs due to the existence of collective aggregation interactions which are stronger among similar particles than for different ones. The consequence of this is the formation of segregated patterns at locations that can be different from one experiment to another. Examples of this are the band segregation in a long drum [5] or stripe formation in an horizontally shaken granular mixture [6].

The origin of these particle-particle interactions can be of different nature, ranging from excluded volume effect $[7,8]$ to long range fluctuation induced forces $[9,10]$. These may appear due to differences of either pressure [11], density [12], or velocity fluctuations [13] in the region between clustering particles and the surroundings. In a previous work [14], we took a well-known system that leads to segregation as it is an horizontally shaken shallow layer of a binary mixture of dry particles [15-20] and we analysed the interaction among pairs of spheres within the bulk of other, different kind of particles. In such conditions, stripe formation has been systematically found in the direction perpendicular to the forcing, a behavior also observed in other systems [21-23]. The particle-particle

\footnotetext{
^e-mail: angel@unav.es
}

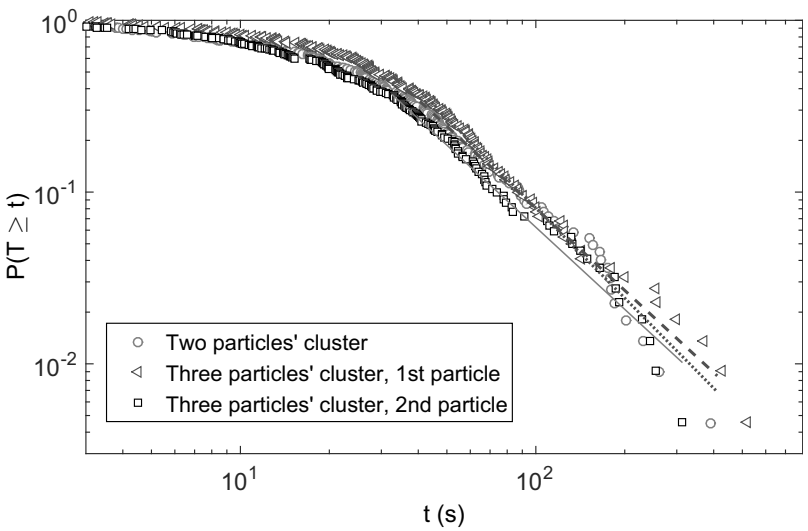

Figure 1. Complementary CDFs of the time that a cluster takes to split. As indicated in the legend the three different symbols indicate 1) the splitting time of a two particles' cluster, 2) the splitting time of the triplet which is given by the time that it takes to the first particle of the cluster to separate from the other two; and 3) the splitting time of the two particles' cluster which remains after the splitting of the triplet. Lines are power law fits of the distribution tails.

interaction study was done by extensive measurements of the dynamics of the pairs of particles when they come together but also by the cluster properties, and the particle separation [14]. The results suggest that the dynamics is controlled by a competition between attraction forces and random agitation, both depending on the packing fraction within the system determined by the amount of bulk particles. As the packing fraction increases, the random motion of the particles reduces and the attraction becomes stronger. Interestingly, even for the densest case (which 


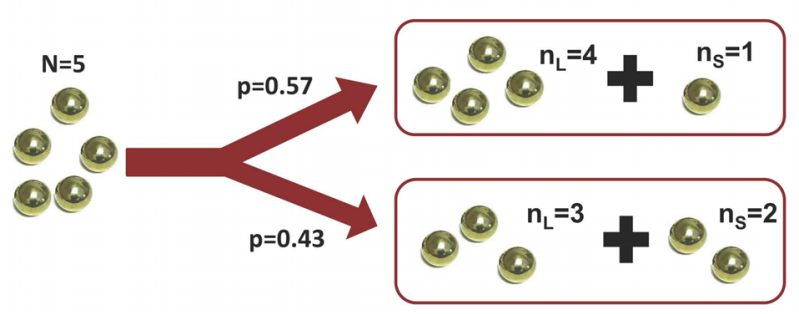

Figure 2. Sketch illustrating the two possible ways in which a 5-particles cluster splits into two smaller ones.

leads to a very segregated pattern) the two particles' clusters eventually split in a finite amount of time. Moreover, it was reported that the distribution of these splitting times is a power law decay, a result that is robust as it does not depend on particle's properties.

\section{The experiment}

Despite all the knowledge gained about particle-particle interactions in our previous work and other recent ones using a similar approach [24-26], its extrapolation to a system with many particles has not been implemented. Obviously, the two particles case represents the foundation upon which to build any theory or model aiming to explain the global segregation. The gap, however, is perhaps still too large and therefore, we believe that the study of the properties of a few particles' clusters could be useful as an intermediate step. This is precisely the goal of this preliminary work where we have studied the splitting process of clusters analysing both the time that it takes to the cluster destruction and the way in which the clusters split in terms of the size of the subclusters produced.

The experimental setup is the same as the one described in [14] in which we are going to work with poppy seeds (kidney shaped, with an average diameter of $d=1 \pm 0.2 \mathrm{~mm}$ ) as bulk particles with a concentration $C=0.91$. Then, we prepare isolated clusters of phosphorbronze spheres $(d=1.5 \mathrm{~mm})$ of size $N$, ranging from $N=2$ to $N=20$ particles. From the initial cluster, we measure the time that it takes until it breaks apart. This condition is fulfilled when any of the particles conforming the cluster separates more than $\Delta-d>12 \mathrm{~mm}$ from the closest one, with $\Delta$ being the distance among the particles centers.

\section{Results}

In Fig. 1 we show the survival time distribution (also called Complementary Cumulative Distribution Function CDF) for a triplet together with the reference case of a duplet in the same experimental conditions. Also, we show the survival distribution of the duplet that remains formed after the separation of the first particle of the triplet. Interestingly, all the distributions look very similar without remarkable differences in the power law exponent $\alpha=2.5$.

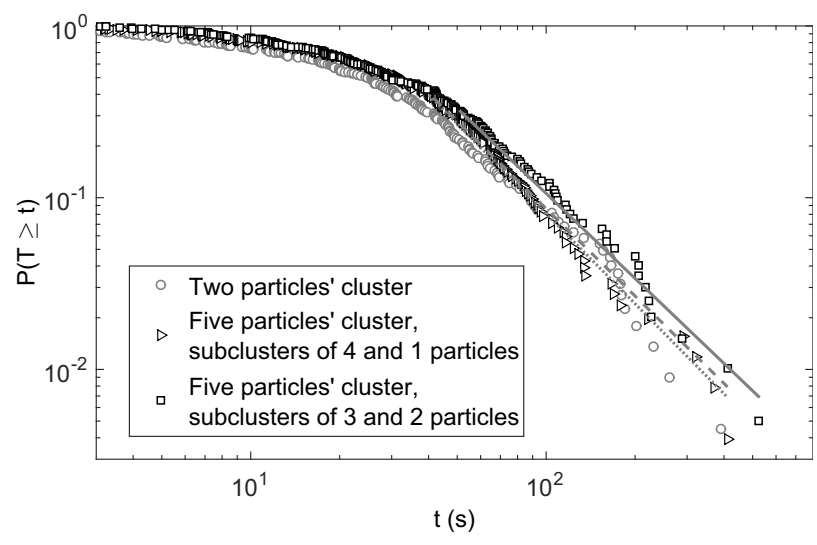

Figure 3. Complementary CDFs of the time that a 5 particles cluster takes to split compared with the case of 2 particles in the same conditions. Two different distributions are shown depending on the way in which the cluster splits: either in two subclusters of 4 and 1 particles or in 3 and 2 as shown in Fig. 2 and indicated in the legend.Lines are power law fits of the distribution tails.

Indeed, the average splitting time of the duplet and the triplet are almost identical: $\langle t\rangle=44 \mathrm{~s}$ for the duplet and $\langle t\rangle=45.8 \mathrm{~s}$ for the triplet. Remark that the notation $\langle t\rangle$ is used in this paper to define an average time. The stability of the duplet remaining after the destruction of the triplet seems to be slightly lower than the standard (isolated) duplet as the distribution shown in Fig. 1 goes systematically below the other ones. In effect, the average splitting time of this remaining duplet is $\langle t\rangle=35.8 \mathrm{~s}$, which is considerably lower than the standard case. This behavior is attributed to the effect that the already escaped particle might have on the other two, causing an effective attraction that helps to the separation of the two particles' remaining cluster.

Independently on what happens with the stability of the remaining cluster, the results obtained for the splitting times of the triplet and the standard duplet prompt us to think that the forces among particles are non-additive. To confirm this, we have performed the same sort of experiments with clusters of $N=5$ particles. In this case, we are not going to look to the dynamics of the remaining clusters as the diversity of situations is rather large. Indeed, there is not a single way of splitting. As sketched in Fig. 2 the remaining subclusters might be in a configuration of $n_{L}=4$ and $n_{S}=1$ or, otherwise, $n_{L}=3$ and $n_{S}=2$, where $n_{L}$ is the number of particles in the larger remaining cluster and $n_{S}$ the number of particles in the smaller one. Note that due to the criteria we use to decide the time at which the cluster has been broken, the remaining clusters at that precise time is necessarily two. Remarkably, the probability of giving rise to a $4+1$ configuration, $p\left(n_{S}=1\right)=0.57$, is larger than the $2+3$ one, $p\left(n_{S}=2\right)=0.43$. Again, for these 5-particles clusters, the survival distribution of the splitting times (Fig. 3) evidences a power law with similar exponents that for the 2 and 3 particles scenario, yet it should be mentioned that the average splitting time increases a bit. 


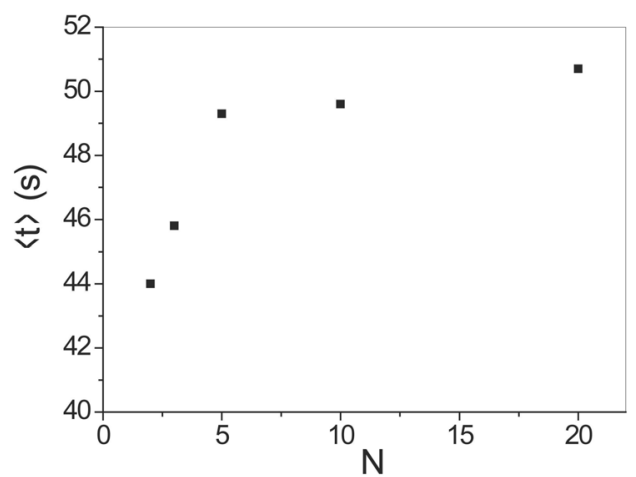

Figure 4. Average splitting time $\langle t\rangle$ of clusters formed by different number of particles $N$.

Having realized the slight tendency to augment the average splitting time with the cluster size, we have performed more experiments with clusters of $N=10$ and $N=20$ particles. The data shown in Fig. 4 suggest that, if this trend exists, it becomes negligible for sufficiently large clusters where the splitting time is independent on the cluster size. This result, which is still not fully understood, definitively shows the non-additive character of the interactions among particles in these experimental conditions.

Finally, we have analysed the way in which the $N=10$ and $N=20$ clusters split in terms of the size of the subclusters remaining after the initial cluster destruction. The outcomes are shown in Fig. $5 \mathrm{a}$ and $5 \mathrm{~b}$ for $N=10$ and $N=20$ respectively. As in the case of 5 particles, the clusters necessarily split in two and the size of the smallest one $\left(n_{S}\right)$ is taken as descriptor. Clearly, clusters break rather asymmetrically, in the sense that the most probable situation is to find a very large and a very small subclusters. This phenomenology was already glimpsed from the results of the 5-particles cluster but it becomes robustly confirmed with the results of these larger clusters. Also, the graphs seem to suggest that the difference between the $19+1$ configuration and $18+2$ is more important than the one between the successive configurations. These results evidence that the way in which clusters break is mostly due to escaping of individual particles from the borders instead of cluster breakage in subclusters of similar size.

Another subtle feature observed in Fig. 5 is that cluster splitting in subclusters of even number of particles is slightly less favoured than splitting in subclusters of odd number of particles. In effect, the probabilities obtained for $n_{S}=2,4$ (in the case of $N=10$ ) and $n_{S}=4,6,8,10$ (in the case of $N=20$ ) are underpopulated when compared with the values that the global trend suggest. We speculate that this phenomenology could be related with a preferred particle arrangement.
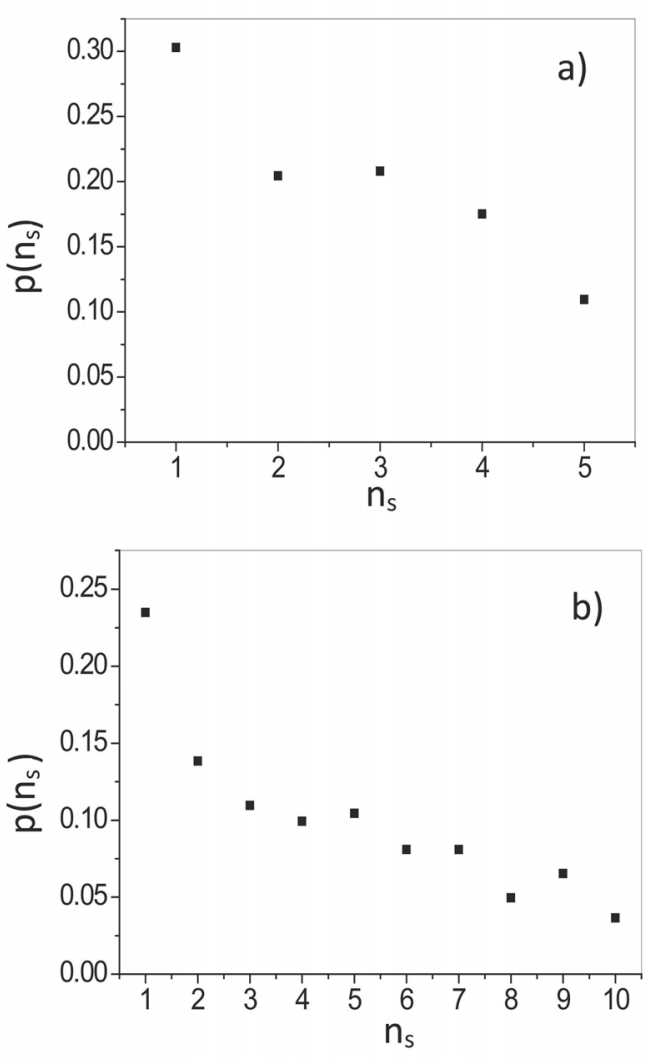

Figure 5. Probability that a cluster of a) $N=10$ and b) $N=20$ particles splits in a configuration where the smaller subcluster is composed of $n_{s}$ particles.

\section{Conclusions}

In this work we have performed a preliminary experimental analysis of the splitting process of clusters of phosphorbronze particles within a sea of poppy seeds. The way in which this study is performed (looking at isolated clusters) supposes a clear differentiation from previous works where the dynamics of patterns with many clusters was investigated, hence observing interactions among clusters as the key factor determining the arising collective behavior. Based on previous knowledge of particle-particle interaction in duplets, we have extended the analysis to many particles' clusters. The first remarkable result is that the time that it takes until any of the particles of the cluster (or a group of them) leaves it does not depend very much on the cluster size. Also it is interesting to see that the clusters tend to split in subclusters of very different size, with a strong tendency to observe a single particle escaping from the other ones. This trend seems to be accentuated when the original cluster size enlarges as the probability of getting $n_{s}=1$ with respect to $n_{s}=2$ increases: it is $32 \%$ higher for $N=5$, for $N=10$ it is $48 \%$ higher, and $70 \%$ higher for $N=20$. From these results it is obvious that further investigation of the geometrical properties of the clusters is necessary. In fact, it is observed that the clusters adopt elongated shapes, mostly perpendicular to the forcing direction. This information, seems to be a crucial 
ingredient to include in any model that aims to reproduce the complexity in the cluster dynamics reported here.

Finally, we would like to mention a collateral result of this work in relation with the stability of the remaining clusters, a measurement that has been only performed for the clusters of three particles. We show that the time that the remaining duplet takes to split is considerably smaller than the one obtained for an isolated duplet. This is attributed to the attraction that the already escaped particle induces on the duplet, hence speeding up the splitting process.

\section{Acknowledgements}

We thank T. Mullin for his help and discussions. This work was funded by Ministerio de Economía y Competitividad (Spanish Government) through Project No. FIS201457325.

\section{References}

[1] I. S. Aranson and L. S. Tsimring, Rev. Mod. Phys. 78, 641 (2006).

[2] A. Kudrolli, Rep. Prog. Phys. 67, 209 (2004).

[3] J. M. Ottino and D. V. Khakhar, Annu. Rev. Fluid Mech. 32, 55 (2000).

[4] M. E. Möbius, B.E. Lauderdale, S.R. Nagel, H.M. Jaeger, Nature 414, 270 (2001).

[5] K. M. Hill, A. Caprihan, and J. Kakalios, Phys. Rev. Lett. 78, 50 (1997).

[6] T. Mullin, Science 295, 1851 (2002).

[7] T. Shinbrot and F. Muzzio, Nature, 410, 251 (2001).

[8] M. Bose, P. R. Nott and V. Kumaran, Europhys. Lett. 68508 (2004).
[9] C. Cattuto, R. Brito, U. M. Marconi, F. Nori, and R. Soto, Phys. Rev. Lett. 96, 178001 (2006).

[10] M. R. Shaebani, J. Sarabadani, and D. E. Wolf, Phys. Rev. Lett. 108, 198001 (2012).

[11] S. Aumaitre, C. A. Kruelle, and I. Rehberg, Phys. Rev. E 64, 041305 (2001).

[12] D. A. Sanders, M. R. Swift, R. M. Bowley, and P. J. King, Phys. Rev. Lett. 93, 208002 (2004).

[13] I. Zuriguel, J. F. Boudet, Y. Amarouchene, and H. Kellay, Phys. Rev. Lett. 95, 258002 (2005).

[14] C. Lozano, I. Zuriguel, A. Garcimartín, and T. Mullin, Phys. Rev. Lett. 114, 178002 (2015).

[15] T. Mullin, Phys. Rev. Lett. 84, 4741 (2000).

[16] P. M. Reis and T. Mullin, Phys. Rev. Lett. 89, 244301 (2002).

[17] P. M. Reis, G. Ehrhardt, A. Stephenson and T. Mullin, Europhys. Lett. 66, 357 (2004).

[18] P. M. Reis, T. Sykes, and T. Mullin, Phys. Rev. E 74, 051306 (2006).

[19] D. Pihler-Puzović and T. Mullin, Proc. Roy. Soc. A 469, 20130203 (2013).

[20] M. P. Ciamarra, A. Coniglio, and M. Nicodemi, Phys. Rev. Lett. 94, 188001 (2005).

[21] H. Löwen, Soft Matter 6, 3133 (2010).

[22] R. Moosavi, M. Maleki, M. Reza Shaebani, J. C. Ruiz-Suárez and E. Clément, Europhys. Lett. 107, 34006 (2014).

[23] R. Möbius and C. Heussinger, Soft Matter 10, 4806 (2014).

[24] G. M. Rodríguez-Liñán, Y. Nahmad-Molinari, G. Pérez-Ángel, PLoS ONE 11(5), e0156153 (2016).

[25] R. A. López de la Cruz and G. A. Caballero-Robledo, Journal of Fluid Mechanics, Volume 800 248-263 (2016).

[26] J. C. Burton, J-J. Liétor-Santos, arXiv:1604.05360v2 